\title{
SYNTHESIS AND CHARACTERIZATION OF NOVEL TRIAZINE COMPOUND AND THEIR BIOLOGICAL STUDIES
}

\author{
Jayesh M. Pandya ${ }^{1}$,, Jyotindra Mahyavanshi ${ }^{1}$ and Smita Bakshi ${ }^{2}$ \\ ${ }^{1}$ Department of Chemistry, Sankalchand Patel University, Visnagar-384315, (Gujarat) India \\ ${ }^{2}$ Department of Botany, Shirmad Rajchandra Vidyapeeth, Dharmpur-396050, (Gujarat) India \\ ${ }^{\square}$ Corresponding Author: jayeshbi1998@yahoo.co.in
}

\begin{abstract}
Novel organic derivatives of 2,4,6 trichloro-1,3,5-triazine (cyanuric chloride) (2) moiety using condensation with various amines to get trisubstituted triazine (3a-h and 4a-h) have been reported. All the newly synthesized compounds are characterized by analytical and spectral tools. The novel synthesized compounds are accessed for their microbial activity.

Keywords: Cyanuric chloride, Simazine, Atrazine, Antimicrobial Activity, Spectral Studies.
\end{abstract}

RASĀYAN J. Chem., Vol. 14, No.2, 2021

\section{INTRODUCTION}

Triazine (1) is a class of agrochemical active ingredients that comprise derivatives having the same backbone with different functionalities to exhibit various herbicidal activities. Simazine, Atrazine, Propazine, Cyanazine are well-known and largely produced herbicide derivatives. These derivatives are helpful for the better growth of crops like Corn and Sugarcane. It also inhibits the growth of unwanted weeds and annual grass. The potential activity of Triazine derivatives is due to various substituent groups attached to a 1,3,5-triazine moiety. Towards the search for new active derivatives of the Triazine class, we wish to report the synthesis and characterization of novel derivatives of Triazine along with their biological activity. Instead of basic Triazine moiety, further derivatization of existing potential herbicides like Simazine and Atrazine has been evaluated. ${ }^{1-2}$

Amongst all Triazine backbones, 1,3,5-Triazine derivatives are more active and extensively studied because of their symmetrical geometry. These Triazine derivatives can be synthesized using Cyanuric acid, melamine and cyanuric chloride as a key starting raw materials. ${ }^{3-5}$

Cyanuric chloride (2) is produced by the trimerization of chlorinated hydrocyanic acid. (Scheme-1) and the prompt reactivity towards nucleophilic aromatic substitution (SNAr) leads us to novel derivatives of the Triazine class. ${ }^{6-9}$

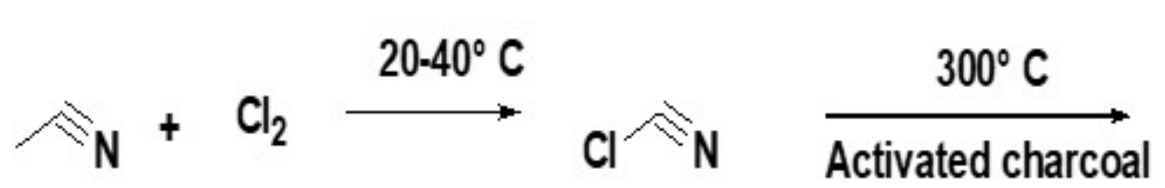<smiles>Clc1nc(Cl)nc(Cl)n1</smiles>

Scheme-1: Industrial Production of Cyanuric Chloride

Numerous reports are available in the literature on the diversity of biological activities of substituted -1,3,5triazine like anti-bacterial, anti-fungal, anti-HIV and other microbial activity. ${ }^{10-18}$

Cyanuric chloride has temperature-oriented differential reactivity for displacement during aromatic nucleophilic substitution reaction. Generally, the first chlorine is a substitute at $0-5^{\circ} \mathrm{C}$, the second chlorine is a substitute at $25-35^{\circ} \mathrm{C}$ and the third chlorine is a substitute at $80-110^{\circ} \mathrm{C}$. This property allows the 
RASĀYAN J. Chem.

Vol. 14 | No. 2 |1183-1190| April - June | 2021

substitution of three different nucleophiles on the same triazine core, which provides a vast variety of possible triazine derivatives and their application. Disubstituted triazines like Simazine and atrazine are a very important herbicide. It is used for preventing pre and postmergerence broadleaf weeds in a crop like maize and sugarcane.

\section{Material and Methods}

\section{EXPERIMENTAL}

Laboratory solvents were purchased from a commercial supplier, Ahmedabad in their pure form. The remaining facilitation was received from Suyog dye chime Pvt. Ltd Ankleshwar. Precoated silica aluminium plates of Merck were used for TLC.

\section{Characterization}

Melting points were determined in open capillary tubes and were uncorrected. The IR spectra were recorded in $\mathrm{KBr}$ pellets on a Shimadzu spectrometer and ${ }^{1} \mathrm{H}$ NMR and ${ }^{13} \mathrm{C}$ NMR spectra were recorded in $\mathrm{CDCl}_{3}$ with TMS as internal standard on a Brucker spectrometer at $400 \mathrm{MHz}$.ESI-MS of selected samples taken on Thermo Scientific Orbitrap Velos ProTM.

\section{General Procedure}

The compound 2, 4, 6-trichloro-1, 3, 5-triazine (cyanuric chloride) is prepared industrially by chlorination of hydrogen cyanide followed by cyclization. This compound is available commercially.

\section{Preparation of N, N'-Diethyl-N"-alkyl-[1,3,5] triazine-2,4,6-triamine (Compound-3 a-h)}

A flowing reaction scheme (Scheme-2) is adopted for the synthesis of N, N'-Diethyl-N"-alkyl$[1,3,5]$ triazine-2,4,6-triamine (compound-3 a-h).<smiles>Clc1nc(Cl)nc(Cl)n1</smiles>

Cyanurlc chloride Mol. Wt.: 184.41

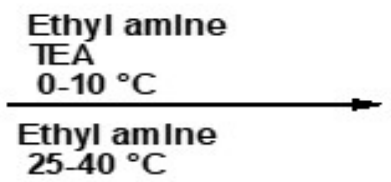

Step-1

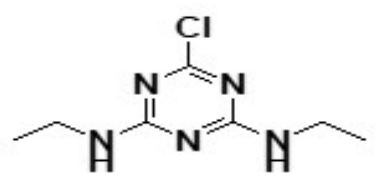

Simazine

$\mathrm{C}_{7} \mathrm{H}_{12} \mathrm{ClN}_{5}$

Mol. Wt.: 201.66

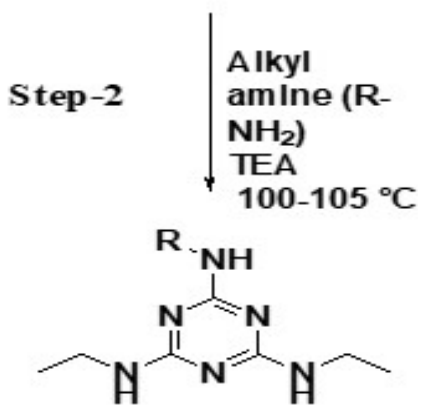

Where, $R-N H 2=$ (a) Propyl amine

(e) 4-Methoxy amine

Compound-3 a-h

(b) Phenylethyl amine

(c) Benzyl amine

(d) Butyl amine

(f) 4-Methoxy phenylethyl amine

(g) 3,4-Di Methoxy phenylethyl amine

(h) 4-Hydrooxy phenylethyl amine

Scheme-2 
RASĀYAN J. Chem.

Vol. 14 | No. 2 |1183-1190| April - June | 2021

Stage-1: Preparation of 6-Chloro-N, N'-diethyl-1,3,5-triazine-2, 4-diamine (Simazine)

Charge $25 \mathrm{gm}$ ( $0.136 \mathrm{~mole})$ of cyanuric chloride and $100 \mathrm{ml}$ of dioxane in $3 \mathrm{~N} \mathrm{RBF}$. Charge $30 \mathrm{gm}(0.297$ mole) of Tri ethyl amine. Cool to $0-5^{\circ} \mathrm{C}$ and stir for 15 minutes. Start addition of 15.5 gm ( 0.34 mole) of ethyl amine through addition funnel. After completion of addition stir reaction mass at $0-10^{\circ} \mathrm{C}$ for $3 \mathrm{hrs}$ then stir it at $30-40^{\circ} \mathrm{C}$ for $6 \mathrm{hrs}$. The reaction is monitored by TLC (Mobile phase: Toluene: Ethyl acetate: 7:3).After completion of reaction dump reaction mixture. stir it for $3 \mathrm{hrs}$ at $25-35^{\circ} \mathrm{C}$. Filter the product and wash with $100 \mathrm{ml}$ water. Dry the product at $70-80 \mathrm{c}$ for $6 \mathrm{hrs}$ in a hot air oven.

\section{Stage-2: Preparation of N, N'-Diethyl-N"-alkyl - [1,3,5]triazine-2,4,6-triamine (Compound-3a-h)} Charge $5 \mathrm{gm}(0.025 \mathrm{~mole})$ of Simazine and $50 \mathrm{ml}$ of dioxane in $3 \mathrm{~N}$ RBF under stirring. Charge $10 \mathrm{gm}(0.1$ mole) of Triethylamine and stir for $10 \mathrm{~min}$. Start addition of alkyl amine (0.03 mole) at 25-35 Slowly raise temperature to $100-105 \mathrm{C}$ and maintain above temperature for $6 \mathrm{hrs}$. The reaction is monitor by TLC (Mobile phase: Toluene: Ethyl acetate::7:3). After completion of reaction cool to 25-35 $\mathrm{c}$ and quench reaction mass in $100 \mathrm{ml}$ water .stir it for $2 \mathrm{hrs}$ and filter it dry it at $60-70 \mathrm{c}$ in hot air oven to get of N, N'-Diethyl-N"alkyl-[1,3,5]triazine-2,4,6-triamine (Compound-3a-h).

\section{Preparation of N-Ethyl-N'-isopropyl-N"'-propyl-[1,3,5] triazine-2,4,6-triamine (compound-4 a-h)}

Flowing reaction scheme (Scheme-3) is adopted for synthesisN-Ethyl-N'-isopropyl-N"-propyl$[1,3,5]$ triazine-2,4,6-triamine (compounds 4a-h).

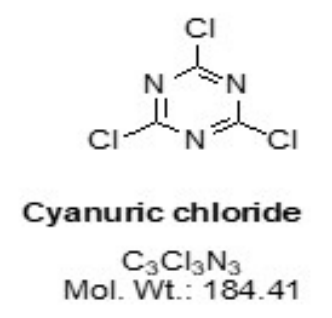

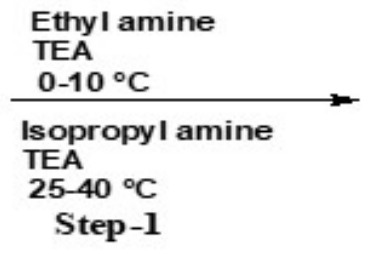

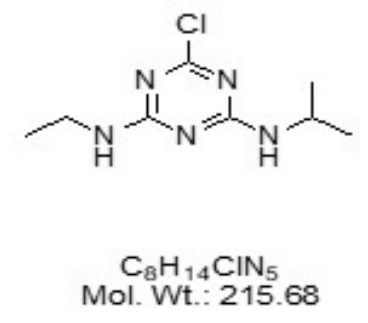

\begin{tabular}{l|l} 
Step-2 & $\begin{array}{l}\text { Alky I amine }\left(\mathrm{R}-\mathrm{NH}_{2}\right) \\
\text { TEA } \\
100-105^{\circ} \mathrm{C}\end{array}$
\end{tabular}<smiles>[R]Nc1nc(NCC)nc(NC(C)C)n1</smiles>

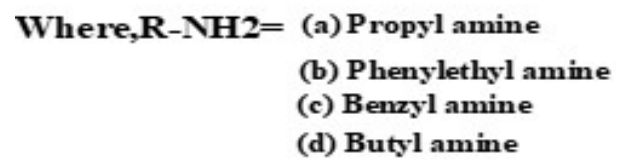

(e) 4-Methoxy amine

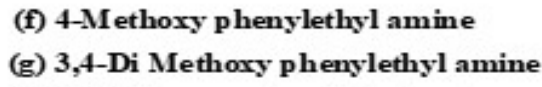

Scheme-3

\section{Stage-1: Preparation of 6-Chloro-N-ethyl N'- Isopropyl -1,3,5-triazine-2,4-diamine (Atrazine)} Charge $25 \mathrm{gm}(0.136 \mathrm{~mole})$ of cyanuric chloride and $100 \mathrm{ml}$ of dioxane in $3 \mathrm{~N} \mathrm{RBF}$. Charge $30 \mathrm{gm}(0.297$ mole) of Triethylamine.Cool to $0-5^{\circ} \mathrm{C}$ and stir for 15 minutes. Start addition of $6.1 \mathrm{gm}(0.136$ mole $)$ of ethyl amine through the addition funnel. After completion of addition stir reaction mass at $0-10 \mathrm{c}$ for $3 \mathrm{hrs}$ then allow coming at $25-35^{\circ} \mathrm{C}$. Start addition of $8 \mathrm{gm}(0.136$ mole $)$ of Isopropyl amine .stirr it at $30-40^{\circ} \mathrm{C}$ for 6 
hrs. The reaction is monitored by TLC (Mobile phase: Toluene: Ethylacetate:: 7:3). After completion of reaction dump reaction mixture. Stir it for $3 \mathrm{hrs}$ at $25-35^{\circ} \mathrm{C}$. filter the product and wash with $100 \mathrm{ml}$ water. Dry the product at $70-80 \mathrm{c}$ for 6 hrs in a hot air oven.

\begin{abstract}
Stage-2: Preparation of N-Ethyl-N'-isopropyl-N"'-alkyl -[1,3,5]triazine-2,4,6-triamine (Compound4a-h)

Charge $5 \mathrm{gm}(0.023 \mathrm{~mole})$ of Atrazine and $50 \mathrm{ml}$ of dioxane in $3 \mathrm{~N}$ RBF under stirring. Charge $5 \mathrm{gm}(0.05$ mole $)$ of Triethylamine and stir for $10 \mathrm{~min}$. Start addition of Alkyl amine $(0.03$ moles $)$ at $25-35{ }^{\circ} \mathrm{C}$ slowly raise temperature to $100-105^{\circ} \mathrm{C}$ and maintain above temperature for $6 \mathrm{hrs}$. The reaction is monitor by TLC (Mobile phase: Toluene: Ethylacetate:: 7:3). After completion to reaction cool to $25-35^{\circ} \mathrm{C}$ and quench reaction mass in $100 \mathrm{ml}$ water, stirr it for $2 \mathrm{hrs}$ and filter it dry it at $60-70 \mathrm{c}$ in hot air oven to get N-EthylN'-isopropyl-N"-alkyl -[1,3,5]triazine-2,4,6-triamine (Compound-4a-h).
\end{abstract}

Yield and Spectroscopic Data of Synthesized Compounds (3a-h and 4a-h)

N, N'-Diethyl-N"'-propyl-[1,3,5] triazine-2,4,6-triamine (Compound-3a)

Yield: 57\%, solid, m.p.93-970 C. M.W.: $224.31 ; \mathrm{m} / \mathrm{z}: 22{ }^{1} \mathrm{H}$ NMR $\left(400 \mathrm{MHz}, \mathrm{CDCl}_{3}\right): \delta$ 0.907-0.956 $(\mathrm{t}, 3 \mathrm{H}), \quad 1.137-1.185 \quad(\mathrm{t}, 6 \mathrm{H}), 1.498-1.615 \quad(\mathrm{t}, 2 \mathrm{H}), 3.293-3.396 \quad(\mathrm{q}, 6 \mathrm{H}), \quad 5.126 \quad(\mathrm{~s}, 1 \mathrm{H}))^{13} \mathrm{C} \quad \mathrm{NMR} \quad(400$

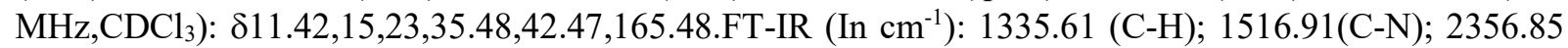
$(\mathrm{C}=\mathrm{N}) ; 2965.35($ Aliphatic $\mathrm{C}-\mathrm{H}) ; 3525.63(\mathrm{~N}-\mathrm{H})$ Anal. Calcd. for $\mathrm{C}_{10} \mathrm{H}_{20} \mathrm{~N}_{6}: \mathrm{C}, 53.55 ; \mathrm{H}, 8.99 ; \mathrm{N}, 37.47$; Found: C, 53.48; H, 8.91; N, 37.41.

N, N'-Diethyl-N"-phenethyl-[1,3,5]triazine-2,4,6-triamine (Compound-3b)

Yield: $61 \%$, solid, m.p.104-107 ${ }^{\circ}$ C. M.W.: $286.38 ; \mathrm{m} / \mathrm{z}: 287^{1} \mathrm{H}$ NMR (400 MHz, $\mathrm{CDCl}_{3}$ ): $\delta 0$ 1.105-1.113

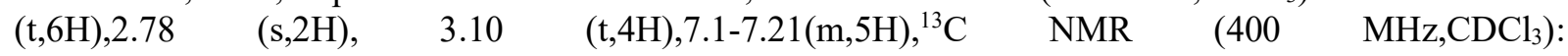
815.5,37.3,44.4,53.7,125.4,127.4,128.6,140.2,175.2,. FT-IR (In $\left.\mathrm{cm}^{-1}\right): 1335.61(\mathrm{C}-\mathrm{H}) ; 1516.91(\mathrm{C}-\mathrm{N})$; $2356.85(\mathrm{C}=\mathrm{N})$; 2965.35(Aliphatic C-H);3030 (Aromatic C-H) 3525.63(N-H), Anal. Calcd. for $\mathrm{C}_{15} \mathrm{H}_{22} \mathrm{~N}_{6}$ : C, 62.91; H, 7.74; N, 29.35; Found: C, 62.83; H, 7.69; N, 29.49.

N, N'-Diethyl-N"-benzyl-[1,3,5]triazine-2,4,6-triamine (Compound-3c)

Yield: $63 \%$, solid, m.p. $114-116^{\circ} \mathrm{C}$. M.W.: 272.35, m/z : $273^{1} \mathrm{H}$ NMR (400 MHz,CDCl $): \delta$ 1.105-1.113 $\begin{array}{lllllll}(\mathrm{t}, 6 \mathrm{H}), & 3.10 & (\mathrm{q}, 4 \mathrm{H}), 4.4 & (\mathrm{~s}, 2 \mathrm{H}) 7.1-7.21(\mathrm{~m}, 5 \mathrm{H}),{ }^{13} \mathrm{C} & \mathrm{NMR} & (400 & \left.\mathrm{MHz}^{2} \mathrm{CDCl}_{3}\right):\end{array}$ 815.5,44.4,57.1,126.5,127.1,128.3,142.2,175.2,. FT-IR (In cm $\left.{ }^{-1}\right): 1330.61(\mathrm{C}-\mathrm{H}) ; 1518.91(\mathrm{C}-\mathrm{N}) ; 2335.85$ $(\mathrm{C}=\mathrm{N})$; 2930.35(Aliphatic $\mathrm{C}-\mathrm{H}) ; 3030($ Aromatic $\mathrm{C}-\mathrm{H}) 3525.63(\mathrm{~N}-\mathrm{H})$, Anal. Calcd. for $\mathrm{C}_{15} \mathrm{H}_{22} \mathrm{~N}_{6}: \mathrm{C}, 61.74$; H, 7.40; N, 30.86; Found: C, 61.68; H, 7.31; N, 30.81 .

N,N'-Diethyl-N"-butyl-[1,3,5] triazine-2,4,6-triamine(Compound-3d)

Yield: $68 \%$, solid, m.p. $122-124^{\circ} \mathrm{C}$. M.W.: $238.33 ; \mathrm{m} / \mathrm{z}: 239^{1} \mathrm{H}$ NMR (400 MHz,CDCl $): \delta$ 1.105-1.113 $(\mathrm{t}, 9 \mathrm{H}), 1.3-1.5(\mathrm{~m}, 4 \mathrm{H}) 3.10-3.20(\mathrm{~m}, 6 \mathrm{H}){ }_{1}^{13} \mathrm{C}$ NMR $\left(400 \mathrm{MHz}, \mathrm{CDCl}_{3}\right): \delta 14,15.5,20.5,34,44.4,51.3,175.2 \mathrm{FT}-$ IR (In cm $\left.{ }^{-1}\right): 1331.61(\mathrm{C}-\mathrm{H}) ; 1518.91(\mathrm{C}-\mathrm{N}) ; 2335.85(\mathrm{C}=\mathrm{N}) ; 2930.35$ (Aliphatic C-H);3030 3525.63(N-H), Anal. Calcd. for $\mathrm{C}_{11} \mathrm{H}_{22} \mathrm{~N}_{6}$ : C, 55.43; H, 9.30; N, 35.26; Found: C, 55.38; H, 9.22; N, 35.21.

N,N'-Diethyl-N"'-(4-methoxy-benzyl)-[1,3,5]triazine-2,4,6-triamine (Compound-3e)

Yield: $72 \%$, solid, m.p.132-136 ${ }^{\circ}$ C. M.W.: 302.37; , m/z: $303^{1} \mathrm{H}_{\mathrm{NMR}}\left(400 \mathrm{MHz}, \mathrm{CDCl}_{3}\right): \delta$ 1.105-1.113

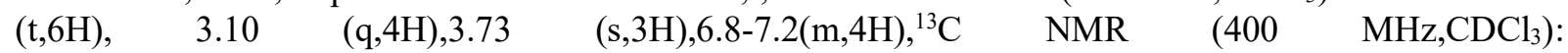
$\delta 15.5,44.4,56,57.2,113.9,128.1,134.7,160,175.2$,. FT-IR (In cm $\left.{ }^{-1}\right): 1320.61$ (C-O); 1518.91(C-N); 2335.85 $(\mathrm{C}=\mathrm{N}) ; 2935.35$ (Aliphatic C-H); 3035 (Aromatic C-H) 3535.63(N-H), Anal. Calcd. for $\mathrm{C}_{15} \mathrm{H}_{22} \mathrm{~N}_{6} \mathrm{O}: \mathrm{C}$, 59.58; H, 7.33; N, 27.79; Found: C, 59.51; H, 7.24; N, 27.71.

N, N'-Diethyl-N"-[2-(4-methoxy-phenyl)-ethyl]-[1, 3, 5] triazine-2,4,6-triamine (Compound-3f) Yield: 69\%, solid, m.p. $141-143^{\circ} \mathrm{C}$. M.W.: $316.40 ; \mathrm{m} / \mathrm{z}: 317,{ }^{1} \mathrm{H}$ NMR (400 MHz,CDCl $): \delta$ 1.105-1.113 $(\mathrm{t}, 6 \mathrm{H}), 2.75-2.8(\mathrm{~m}, 2 \mathrm{H}) 3.10(\mathrm{q}, 4 \mathrm{H}), 3.35-3.4(\mathrm{t}, 2 \mathrm{H}) 3.73(\mathrm{~s}, 3 \mathrm{H}), 6.8-7.1(\mathrm{~m}, 4 \mathrm{H}),{ }^{13} \mathrm{C}$ NMR $\left(400 \mathrm{MHz}, \mathrm{CDCl}_{3}\right)$ : 
815.5,37.3,44.4,53.7,56,113.9,128.3,132.7,159.4,175.2,. FT-IR (In cm $\left.{ }^{-1}\right)$ : 1322.61 (C-O); 1518.91(C-N); $2335.85(\mathrm{C}=\mathrm{N})$; 2935.35(Aliphatic $\mathrm{C}-\mathrm{H}$ ); 3035 (Aromatic $\mathrm{C}-\mathrm{H}$ ) 3535.63(N-H), Anal. Calcd. for $\mathrm{C}_{15} \mathrm{H}_{22} \mathrm{~N}_{6} \mathrm{O}: \mathrm{C}, 60.74 ; \mathrm{H}, 7.65 ; \mathrm{N}, 26.56$; Found: $\mathrm{C}, 60.68 ; \mathrm{H}, 7.59 ; \mathrm{N}, 26.51$.

\begin{abstract}
N, N'-Diethyl-N"'-[2-(3,4-dimethoxy-phenyl)-ethyl]-[1,3,5]triazine-2,4,6-triamine (Compound-3g) Yield: $61 \%$, solid, m.p. $121-127^{\circ} \mathrm{C}$. M.W.: $346.4 ; \mathrm{m} / \mathrm{z}: 347,{ }^{1} \mathrm{H}$ NMR $\left(400 \mathrm{MHz}, \mathrm{CDCl}_{3}\right): \delta$ 1.105-1.113 $(\mathrm{t}, 6 \mathrm{H}), 2.75-2.8 \quad(\mathrm{~m}, 2 \mathrm{H}) \quad 3.10 \quad(\mathrm{q}, 4 \mathrm{H}), 3.73 \quad(\mathrm{~s}, 6 \mathrm{H}), 6.6-6.8 \quad(\mathrm{~m}, 3 \mathrm{H}),{ }^{13} \mathrm{C} \quad \mathrm{NMR} \quad\left(400 \quad \mathrm{MHz}, \mathrm{CDCl}_{3}\right)$ : 815.5,37.4,44.4,53.7,56.3,114.5,115,121.2,133.4,144.8,147.5,175.2,. FT-IR (In $\mathrm{cm}^{-1}$ ): 1325.61 (C-O); 1518.91(C-N); $2335.85(\mathrm{C}=\mathrm{N})$; 2935.35(Aliphatic C-H); $3035($ Aromatic $\mathrm{C}-\mathrm{H})$ 3535.63(N-H), Anal. Calcd. for $\mathrm{C}_{16} \mathrm{H}_{24} \mathrm{~N}_{6} \mathrm{O}_{2}: \mathrm{C}, 57.81 ; \mathrm{H}, 7.28 ; \mathrm{N}, 25.28$; Found: C, 57.74; H, 7.21; N, 25.21.
\end{abstract}

\title{
N,N'-Diethyl-N"'-[2-(4-hydroxy-phenyl)-ethyl]-[1,3,5]triazine-2,4,6-triamine (Compound-3h)
}

Yield: $63 \%$, solid, m.p. $139-143^{0} \mathrm{C}$. M.W.: $302.37 ; \mathrm{m} / \mathrm{z}: 303,{ }^{1} \mathrm{H}$ NMR $\left(400 \mathrm{MHz}, \mathrm{CDCl}_{3}\right): \delta$ 1.105-1.113 $(\mathrm{t}, 6 \mathrm{H}), 2.75-2.8 \quad(\mathrm{~m}, 2 \mathrm{H}) \quad 3.10 \quad(\mathrm{q}, 4 \mathrm{H}), 3.37-3.40(\mathrm{t}, 2 \mathrm{H}), 6.7-7.01 \quad(\mathrm{~m}, 4 \mathrm{H}),{ }^{13} \mathrm{C} \quad \mathrm{NMR} \quad\left(400 \mathrm{MHz}, \mathrm{CDCl}_{3}\right)$ : $\delta 15.5,37.4,44.4,53.7,115.6,129.3,132.8,175.2$,. FT-IR $\left(\mathrm{In} \mathrm{cm}^{-1}\right): 1320.5$ (C-O); 1516.91(C-N); 2356.85 $(\mathrm{C}=\mathrm{N}) ; 2$ 2965.35(Aliphatic C-H);3035 (Aromatic C-H) 3525.63(N-H),3610 (O-H), Anal. Calcd. for $\mathrm{C}_{15} \mathrm{H}_{22} \mathrm{~N}_{6} \mathrm{O}$ : C, 59.58; H, 7.33; N, 27.79; Found: C, 59.51; H, 7.27; N, 27.71.

\section{N-Ethyl-N'-isopropyl-N"'-propyl-[1,3,5]triazine-2,4,6-triamine (Compound-4a)}

Yield: 70\%, solid, m.p. $97-99^{\circ} \mathrm{C}$. M.W.: 238.33 ; m/z : $239{ }^{1} \mathrm{H}$ NMR $\left(400 \mathrm{MHz}, \mathrm{CDCl}_{3}\right): \delta$ 0.916--0.965 $(\mathrm{t}, 3 \mathrm{H}), 1.145-1.191(\mathrm{~d}, 6 \mathrm{H}), 1.504-1.625(\mathrm{t}, 3 \mathrm{H}), 2.332(\mathrm{q}, 4 \mathrm{H}), 3.270-3.419(\mathrm{sq}, 1 \mathrm{H}) 4.092-4.156(\mathrm{~b}, 1 \mathrm{H}), 4.726$

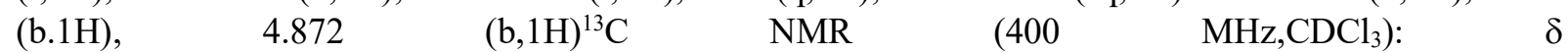
11.43,15.04,22.94,23.04,35.48,42.11,42.45,165.05,165.82.FT-IR ( In cm $\left.^{-1}\right): 1335.61(\mathrm{C}-\mathrm{H}) ; 1515.94(\mathrm{C}-\mathrm{N})$; 2964.39 (Aliphatic C-H); $3311.55(\mathrm{~N}-\mathrm{H})$, Anal. Calcd. for $\mathrm{C}_{11} \mathrm{H}_{22} \mathrm{~N}_{6}$ : C, 55.43; H, 9.30; N, 35.26; Found: C, 55.38; H, 9.22; N, 35.21.

\section{N-Ethyl-N'-isopropyl-N"'-phenethyl-[1,3,5]triazine-2,4,6-triamine (Compound-4b)}

Yield: $63 \%$, solid, m.p.105-108 ${ }^{\circ}$ C. M.W.: $300.40 ; \mathrm{m} / \mathrm{z}: 301^{1} \mathrm{H}$ NMR $\left(400 \mathrm{MHz}, \mathrm{CDCl}_{3}\right): \delta$ 0.934-0.965 $(\mathrm{t}, 3 \mathrm{H}), 1.112-1.137(\mathrm{~d}, 6 \mathrm{H}), 2.78(\mathrm{t}, 2 \mathrm{H}), 3.10(\mathrm{q}, 2 \mathrm{H}) 3.270-3.419(\mathrm{sq}, 1 \mathrm{H}), 3.4(\mathrm{t}, 2 \mathrm{H}), 7.08(\mathrm{t}, 1 \mathrm{H})$ 7.12-7.22 $(\mathrm{d}, 4 \mathrm{H}){ }^{13} \mathrm{C} \quad \mathrm{NMR} \quad\left(400 \quad \mathrm{MHz}, \mathrm{CDCl}_{3}\right): \quad \delta 15.5,23.8,37.3,44.4,49.8, \quad 53.7,125.7, \quad 127.9$, 128.4,140.2,165.05,165.82,166.2 FT-IR (In cm $\left.{ }^{-1}\right)$ : $1335.61(\mathrm{C}-\mathrm{H}) ; 1515.94$ (C-N); 2964.39 (Aliphatic CH);3020 (Aromatic C-H) $3311.55(\mathrm{~N}-\mathrm{H})$, Anal. Calcd. for $\mathrm{C}_{16} \mathrm{H}_{24} \mathrm{~N}_{6}$ : C, 63.97; H, 8.05; N, 27.98; Found: C, 63.91; H, 7.99; N, 27.92.

\section{N-Benzyl-N'-ethyl-N"-isopropyl-[1,3,5]triazine-2,4,6-triamine(Compound-4c)}

Yield: $67 \%$, solid, m.p. $111-113^{0} \mathrm{C}$. M.W.: 286.38;m/z : $287{ }^{1} \mathrm{H}$ NMR $\left(400 \mathrm{MHz}, \mathrm{CDCl}_{3}\right): \delta$ 0.934-0.965 $(\mathrm{t}, 3 \mathrm{H}), 1.102-1.117(\mathrm{~d}, 6 \mathrm{H}), 3.10(\mathrm{q}, 2 \mathrm{H}) 3.270-3.419(\mathrm{sq}, 1 \mathrm{H}), 4.32(\mathrm{~s}, 2 \mathrm{H}) 7.08-7.14(\mathrm{~m}, 5 \mathrm{H}){ }^{13} \mathrm{C}$ NMR $(400$

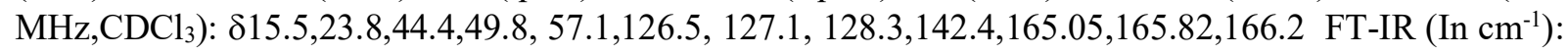
$1335.61(\mathrm{C}-\mathrm{H}) ; 1515.94(\mathrm{C}-\mathrm{N}) ; 2964.39$ (Aliphatic C-H);3022 (Aromatic C-H) $3311.55(\mathrm{~N}-\mathrm{H})$, Anal. Calcd. for $\mathrm{C}_{15} \mathrm{H}_{22} \mathrm{~N}_{6}$ : C, 62.91; H, 7.74; N, 29.35; Found: C, 62.89; H, 7.69; N, 29.32.

\section{N-Butyl-N'-ethyl-N"'-isopropyl-[1,3,5]triazine-2,4,6-triamine(Compound-4d) \\ Yield: $74 \%$, solid, m.p. $118-121^{\circ} \mathrm{C}$. M.W.: 252.36;m/z : $253{ }^{1} \mathrm{H}$ NMR $\left(400 \mathrm{MHz}, \mathrm{CDCl}_{3}\right): \delta$ 0.934-0.965 $(\mathrm{t}, 6 \mathrm{H}), 1.102-1.117(\mathrm{~d}, 6 \mathrm{H}), 1.30-1.33(\mathrm{~m}, 2 \mathrm{H}), 1.50-1.52(\mathrm{t}, 2 \mathrm{H}) 3.10(\mathrm{q}, 2 \mathrm{H}) 3.270-3.419(\mathrm{sq}, 1 \mathrm{H}), 4.32(\mathrm{~s}, 2 \mathrm{H})$

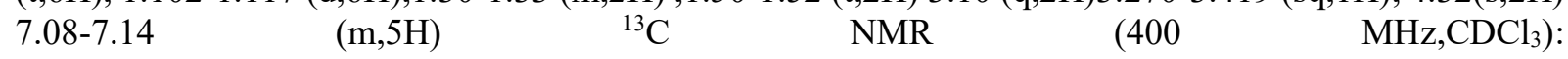 813.5, 15.5,20.5,23.8,31.8,44.4,49.8,51.3,165.05,165.82,166.2 FT-IR (In $\left.\mathrm{cm}^{-1}\right)$ : $1335.61(\mathrm{C}-\mathrm{H}) ; 1515.94$ (C-N); 2964.39 (Aliphatic C-H), $3311.55(\mathrm{~N}-\mathrm{H})$, Anal. Calcd. for $\mathrm{C}_{12} \mathrm{H}_{24} \mathrm{~N}_{6}$ : C, 57.11; H, 9.59; N, 33.30; Found: C, 57.08; H, 9.51; N, 35.21.}

\section{N-Ethyl-N'-isopropyl-N"-(4-methoxy-benzyl)-[1,3,5] triazine-2,4,6-triamine (Compound-4e)}

Yield: $72 \%$, solid, m.p. $131-133^{\circ} \mathrm{C}$. M.W.: $316.40 ; \mathrm{m} / \mathrm{z}: 317{ }^{1} \mathrm{H}$ NMR $\left(400 \mathrm{MHz}, \mathrm{CDCl}_{3}\right): \delta$ 0.934-0.965 $(\mathrm{t}, 3 \mathrm{H}), 1.102-1.117(\mathrm{~d}, 6 \mathrm{H}), 3.10(\mathrm{q}, 2 \mathrm{H}) 3.270-3.419(\mathrm{sq}, 1 \mathrm{H}), 3.73(\mathrm{~s}, 3 \mathrm{H}), 4.32(\mathrm{~s}, 2 \mathrm{H}) 6.8-7.02(\mathrm{~m}, 4 \mathrm{H}){ }^{13} \mathrm{C}$ 
RASĀYAN J. Chem.

Vol. 14 | No. 2 |1183-1190| April - June | 2021

NMR (400 MHz, $\left.\mathrm{CDCl}_{3}\right): \delta 15.5,23.8,44.4,49.8,56.1,57.1,113.9,128.1,134.7,154.7,165.05,165.82,166.2$ FT-IR (In cm $\left.{ }^{-1}\right)$ : 1320.61 (C-O); $1518.91 \quad(\mathrm{C}-\mathrm{N}), 2935.35$ (Aliphatic C-H); 3035 (Aromatic C-H) 3535.63(N-H), Anal. Calcd. for $\mathrm{C}_{12} \mathrm{H}_{24} \mathrm{~N}_{6}$ : C, 60.74; H, 7.65; N, 26.56; Found: C, 60.68; H, 7.62; N, 26.52 .

N-Ethyl-N'-isopropyl-N"-[2-(4-methoxy-phenyl)-ethyl]-[1,3,5]triazine-2,4,6-triamine(Compound-4f) Yield: $61 \%$, solid, m.p. $139-141^{\circ} \mathrm{C}$. M.W.: $330.43 ; \mathrm{m} / \mathrm{z}: 330.2^{1} \mathrm{H}$ NMR $\left(400 \mathrm{MHz}, \mathrm{CDCl}_{3}\right): \delta$ 0.934-0.965 $(\mathrm{t}, 3 \mathrm{H}), 1.102-1.117(\mathrm{~d}, 6 \mathrm{H}), 2.76-2.79(\mathrm{t}, 2 \mathrm{H}) 3.10(\mathrm{q}, 2 \mathrm{H}), 3.39(\mathrm{t}, 2 \mathrm{H}) 3.270-3.419(\mathrm{sq}, 1 \mathrm{H}), 3.73(\mathrm{~s}, 3 \mathrm{H}), 6.8-$ $7.02 \quad(\mathrm{~m}, 4 \mathrm{H}) \quad{ }^{13} \mathrm{C} \quad \mathrm{NMR} \quad\left(400 \quad \mathrm{MHz}, \mathrm{CDCl}_{3}\right): \quad \delta 15.5,23.8, \quad 37.3, \quad 44.4,56, \quad 114,128.9,132.5$, 165.05,165.82,166.2.FT-IR (In cm ${ }^{-1}$ ): 1320.61 (C-O); $1518.91 \quad$ (C-N), 2935.35(Aliphatic C-H); 3035 (Aromatic C-H) 3535.63(N-H), Anal. Calcd. for $\mathrm{C}_{17} \mathrm{H}_{26} \mathrm{~N}_{6} \mathrm{O}: \mathrm{C}, 61.79 ; \mathrm{H}, 7.93$; N, 25.43; Found: C, 61.72; $\mathrm{H}, 7.87 ; \mathrm{N}, 25.41$.

\section{N-[2-(3,4-Dimethoxy-phenyl)-ethyl]-N'-ethyl-N"'-isopropyl-[1,3,5]triazine-2,4,6- triamine(Compound-4g)}

Yield: $67 \%$, solid, m.p. $118-122^{\circ} \mathrm{C}$. M.W.: $360.45 ; \mathrm{m} / \mathrm{z}: 361^{1} \mathrm{H}$ NMR $\left(400 \mathrm{MHz}, \mathrm{CDCl}_{3}\right): \delta$ 0.934-0.965 $(\mathrm{t}, 3 \mathrm{H}), 1.102-1.117(\mathrm{~d}, 6 \mathrm{H}), 2.76-2.79(\mathrm{t}, 2 \mathrm{H}) 3.10(\mathrm{q}, 2 \mathrm{H}), 3.39(\mathrm{t}, 2 \mathrm{H}) 3.270-3.419(\mathrm{sq}, 1 \mathrm{H}), 3.73(\mathrm{~s}, 6 \mathrm{H}), 6.8-$ $7.02(\mathrm{~m}, 3 \mathrm{H}){ }^{13} \mathrm{C}$ NMR $\left(400 \mathrm{MHz}, \mathrm{CDCl}_{3}\right): \delta 15.5,23.8,37.6,44.4,49.8,53.7,56.3,114.5,115,121.2,133.6$, 165.05,165.82,166.2 FT-IR (In cm ${ }^{-1}$ ): 1320.61 (C-O); $1518.91 \quad$ (C-N), 2935.35(Aliphatic C-H); 3035 (Aromatic C-H) 3535.63(N-H), Anal. Calcd. for $\mathrm{C}_{18} \mathrm{H}_{28} \mathrm{~N}_{6} \mathrm{O}_{2}: \mathrm{C}, 59.98 ; \mathrm{H}, 7.83$; N, 23.32; Found: C, 59.91; H, 7.73; N, 23.31 .

\section{4-[2-(4-Ethylamino-6-isopropylamino-[1,3,5] triazin-2-ylamino)-ethyl]-phenol (Compound-4h)}

Yield: $70 \%$, solid, m.p. $138-142^{\circ} \mathrm{C}$. M.W.: $316.40 ; \mathrm{m} / \mathrm{z}: 317,{ }^{1} \mathrm{H}$ NMR $\left(400 \mathrm{MHz}, \mathrm{CDCl}_{3}\right): \delta$ 0.934-0.965 $(\mathrm{t}, 3 \mathrm{H}), 1.102-1.117(\mathrm{~d}, 6 \mathrm{H}), 2.76-2.79(\mathrm{t}, 2 \mathrm{H}) 3.10(\mathrm{q}, 2 \mathrm{H}), 3.39(\mathrm{t}, 2 \mathrm{H}) 3.270-3.419(\mathrm{sq}, 1 \mathrm{H}), 6.72-6.85(\mathrm{~m}, 4 \mathrm{H})$ ${ }^{13} \mathrm{C} \quad \mathrm{NMR} \quad\left(400 \quad \mathrm{MHz}, \mathrm{CDCl}_{3}\right): \quad \delta 15.5,23.8, \quad 37.6, \quad 44.4,49.8,53.7,56.3, \quad 115.6,129.3,132.8,154.5$, 165.05,165.82,166.2 FT-IR (In cm $\left.{ }^{-1}\right): 1335.61(\mathrm{C}-\mathrm{H}) ; 1515.94(\mathrm{C}-\mathrm{N}) ; 2964.39$ (Aliphatic C-H); 3020 (Aromatic C-H),3311.55 (N-H), Anal. Calcd. for $\mathrm{C}_{16} \mathrm{H}_{24} \mathrm{~N}_{6} \mathrm{O}$ : C, 60.74; H, 7.65; N, 26.56; Found: C, 60.68; H, 7.58; N, 26.51 .

\section{Biological Screening}

\section{Antibacterial Activity}

Antibacterial activities of all the compounds were studied against gram-positive bacteria (Staphyloco) and gram-negative bacteria (E.coli, and p.aeruginosa) at a concentration of $50 \mu \mathrm{g} / \mathrm{ml}$ by agar cup plate method. The methanol system was used as a control in this method. Under similar conditions using streptomycin as a standard for comparison carried out control experiment. The area of inhibition of zone measured in $\mathrm{mm}$. and found value is tabulated in Table (Tabel-1).

Table-1: Antibacterial Activity of Compounds (3a-h and 4a-h)

\begin{tabular}{c|c|c|c|c}
\hline \multirow{2}{*}{ Compound } & \multicolumn{4}{|c}{$\begin{array}{c}\text { Minimal Inhibition Concentration of } \\
\text { Bacterial Strains (MIC) in } \mu \mathrm{g} / \mathrm{ml}\end{array}$} \\
\cline { 2 - 5 } & Gram Negative Strains & Gram Positive Strains \\
\cline { 2 - 5 } & $\begin{array}{c}\text { E. coli } \\
\text { MTCC-443 }\end{array}$ & $\begin{array}{c}\text { P. aeruginosa } \\
\text { MTCC-1688 }\end{array}$ & $\begin{array}{c}\text { B. subtilis } \\
\text { MTCC-441 }\end{array}$ & $\begin{array}{c}\text { S.aureus } \\
\text { MTCC-96 }\end{array}$ \\
\hline 3a & 1.0 & 8.0 & 16.0 & 16.0 \\
\hline 3b & 4.0 & 4.0 & 16.0 & 32.0 \\
\hline $3 \mathrm{c}$ & 0.5 & 8.0 & 16.0 & 16.0 \\
\hline $3 \mathrm{~d}$ & 4.0 & 8.0 & 32.0 & 16.0 \\
\hline $3 \mathrm{e}$ & 4.0 & 8.0 & 4.0 & 16.0 \\
\hline $3 \mathrm{f}$ & 4.0 & 4.0 & 8.0 & 8.0 \\
\hline $3 \mathrm{~g}$ & 2.0 & 2.0 & 8.0 & 4.0 \\
\hline 3h & 8.0 & 4.0 & 4.0 & 16.0 \\
\hline 4a & 4.0 & 4.0 & 8.0 & 2.0 \\
\hline 4b & 1.0 & 1.0 & 2.0 & 8.0 \\
\hline $4 \mathrm{c}$ & 8.0 & 8.0 & 16.0 & \\
\hline
\end{tabular}


RASĀYAN J. Chem.

Vol. 14 | No. 2 |1183-1190| April - June | 2021

\begin{tabular}{c|c|c|c|c}
\hline $4 \mathrm{~d}$ & 2.0 & 2.0 & 8.0 & 4.0 \\
\hline $4 \mathrm{e}$ & 4.0 & 8.0 & 4.0 & 0.8 \\
\hline $4 \mathrm{f}$ & 4.0 & 4.0 & 8.0 & 4.0 \\
\hline $4 \mathrm{~g}$ & 1.0 & 1.0 & 8.0 & 4.0 \\
\hline $4 \mathrm{~h}$ & 4.0 & 8.0 & 8.0 & 16.0 \\
\hline Streptomycin & $\mathbf{0 . 2 5}$ & $\mathbf{0 . 5}$ & $\mathbf{0 . 2 5}$ & $\mathbf{0 . 2 5}$ \\
\hline
\end{tabular}

\section{Antifungal Activity}

The fungicidal activity of all the compounds was studied at $1000 \mathrm{ppm}$ concentration in vitro. Plant pathogenic organisms used were (Aspergillumsniger) The antifungal activity of all the compounds (3a-h and 4-h) was measured and tabulated in the table (Tabel-2).

\begin{tabular}{|c|c|}
\hline \multirow{3}{*}{ Compound } & $\begin{array}{c}\text { Minimal Inhibition } \\
\text { Concentration of Bacterial } \\
\text { Strains (MIC) in } \mu \mathrm{g} / \mathrm{ml}\end{array}$ \\
\hline & Fungal Strains \\
\hline & $\begin{array}{c}\text { A. niger } \\
\text { MTCC-282 }\end{array}$ \\
\hline $3 a$ & 256.0 \\
\hline $3 b$ & 128.0 \\
\hline $3 c$ & 512.0 \\
\hline $3 d$ & 128.0 \\
\hline $3 e$ & 512.0 \\
\hline $3 f$ & 128.0 \\
\hline $3 g$ & 256.0 \\
\hline $3 \mathrm{~h}$ & 256.0 \\
\hline $4 a$ & 512.0 \\
\hline $4 \mathrm{~b}$ & 64.0 \\
\hline $4 \mathrm{c}$ & 512.0 \\
\hline $4 d$ & 256.0 \\
\hline $4 \mathrm{e}$ & 128.0 \\
\hline $4 \mathrm{f}$ & 256.0 \\
\hline $4 \mathrm{~g}$ & 64.0 \\
\hline $4 \mathrm{~h}$ & 64.0 \\
\hline Kanamycin & 4.0 \\
\hline
\end{tabular}

RESULTS AND DISCUSSION

It was observed that 2,4,6 -trichloro 1,3,5-triazine (1) on condensation with a various amine to get tri substituted triazine (compound $3 \mathrm{a}-\mathrm{h}$ and $4 \mathrm{a}-\mathrm{h}$ ). The is confirmed by various analysis like elemental analysis and spectroscopical analysis (ESI-MS,1H NMR, 13 C NMR and IR). The C, H, N analysis and ${ }^{1} \mathrm{H}-\mathrm{NMR}$ data of all compounds are presented above.

\section{ACKNOWLEDGEMENT}

The authors are thankful to the Head, Department of Chemistry, Smt S.S. Patel Nootan Science and Commerce CollegeVisnagar for providing laboratory facilities. The authors wish to extend their gratitude towards the Directors of Suyog Dye cheime for providing valuable support during the entire research.

\section{REFERENCES}

1. F. Ackerman, International Journal of Occupational and Environmental Health, 13(4),437(2007), DOI: 10.1179/oeh.2007.13.4.437

2. C. J. Swanton, R. H.Gulden, K.Chandler, Weed Science, 55(1), 75(2007), DOI:10.1614/WS-06-104.1

3. E. M. Smolin, L. Rapoport, Angewandte Chemie,72(16),601(1960), DOI:10.1002/ange.19600721616

4. H. Neunhoeffer, P. F.Wiley, Chemistry of 1,2,3-Triazines and 1,2,4-Triazines,Tetrazines, and Pentazines, 33, 1335 (1978) 
RASĀYAN J. Chem.

Vol. 14 | No. 2 |1183-1190| April - June | 2021

5. J. M. E. Quirke, Comprehensive Heterocyclic Chemistry, 3(2B), 457(1984), DOI: $10.1002 /$ jps.2600740232

6. E. Havrankova, J. Csollei, P. Pazdera, Molecules, 24,3586(2019), DOI:10.3390/molecules24193586

7. G. Giacomelli, A. Porcheddu, L. De Luca, Current Organic Chemistry, 8, 1497(2004), DOI: $10.2174 / 1385272043369845$

8. T. Eicher, S. Hauptmann, A. Speicher, The Chemistry of Heterocycles-Structures, Reactions, Synthesis, and Applications, pp.556(2003)

9. H. Gröger, J. Sans, T. Güthner, Chimica Oggi/ChemistryToday, 18(3/4), 12(2000)

10. V. Sareen, V. Khatri, P. Jain, Indian Journal of Chemistry, 45B,1288 (2006)

11. K. Prabhakara, Bioinformation, 6(2), 74(2011), DOI:10.6026/97320630006074

12. A. Baliani, M. Stewart, R. Brun, Journal of Medicinal Chemistry, 48,5570(2005), DOI: $10.1021 / \mathrm{jm} 050177+$

13. F. Aczewski, A. Bulakowska, P. Bednarski, R. Gruner, European Journal of Medicinal Chemistry, 41,219(2006), DOI:10.1016/j.ejmech.2006.09.019

14. D. Wall, Bioorganic \& Medicinal Chemistry Letters, 16, 5451(2006), DOI:10.1016/j.bmcl.2006.07.052

15. C. A. M. Afonso, N. M. T. Lourenco, D. Androia, A. Rosatella, Molecules, 11,81(2006), DOI:10.3390/ 11010081

16. K. H. Chikhaliya, R. B. Patel, C. Pannecouque, E. D. Clercq, Journal of the Brazilian Chemical Society, 18(2),312(2007), DOI:10.1590/S0103-50532007000200011

17. P. Gahtori, B. K. Singh, A. Das, Dhaka University Journal of Pharmaceutical Sciences,7(2),107(2008), DOI:10.3329/dujps.v7i2.2164

18. V. G. M. Naidu, Medicinal Chemistry Research, 24, 3991(2015), DOI:10.1007/s00044-015-1430-9

[RJC-6277/2020] 